\title{
Relaxation of Free Volume in Polycarbonate and Polystyrene Studied by Positron Annihilation Lifetime Spectroscopy
}

\author{
D. Cangialosi ${ }^{a, *}$, M. WÜBbenhorst ${ }^{a}, \mathrm{H} . \mathrm{Schut}^{b}$ \\ AND S.J. PICKEN ${ }^{a}$ \\ ${ }^{a}$ Dept. of Polymer Materials and Engineering, Delft University of Technology \\ Julianalaan 136, 2628 BL, Delft, The Netherlands \\ ${ }^{b}$ Interfaculty Reactor Institute, Delft University of Technology \\ Mekelweg 15, 2629 JB, Delft, The Netherlands
}

\begin{abstract}
We present the time evolution of polycarbonate and polystyrene free volume probed by positron annihilation lifetime spectroscopy, which occurs after thermal rejuvenation above the glass transition temperature $\left(T_{\mathrm{g}}\right)$ and after plastic deformation via cold rolling. The ortho-positronium $\left(\tau_{o-\mathrm{Ps}}\right)$

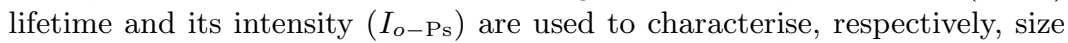
and concentration of the free volume holes. Our results suggest that the effect of plastic deformation on the polymer microstructure is far different from that of a thermally rejuvenated sample contrarily to common beliefs. In particular, plastic deformation results in an increase in $\tau_{o-P s}$ and a decrease in $I_{o-\mathrm{Ps}}$, whereas rejuvenation above $T_{\mathrm{g}}$ results in an increase in $I_{o-\mathrm{Ps}}$ while $\tau_{o-P s}$ remains unchanged. In addition, physical aging below $T_{\mathrm{g}}$ after plastic deformation manifests as a reduction of $\tau_{o-\mathrm{Ps}}$, whereas a decrease in $I_{o-\mathrm{Ps}}$ is observed after thermal rejuvenation. These results corroborate the idea of an amorphous-amorphous transition induced by plastic deformation.
\end{abstract}

PACS numbers: 78.70.Bj, 82.35.Lr, 64.70.Kb

\section{Introduction}

The search for a link between mobility in the glassy state and thermodynamics has always been a challenge in the physics of glass forming liquids. In this framework, theories relating mobility and free volume of glasses have been pro-

*Present address: Fundacion Donostia International Physics Center, Paseo Manuel de Lardizabal, 4-20018 San Sebastian, Spain 
posed in the past by Cohen and Turnbull [1], who suggested that the amount of free space determines the mobility in the glassy state. Later Kovacs [2] and Struik [3] modelled physical aging of polymer glasses, i.e. the slow evolution of thermodynamics properties which generally occurs in glass formers after cooling from above the glass temperature $\left(T_{\mathrm{g}}\right)$, according to this idea. In particular, they described physical aging as a self-retarding process, where the reduction of free volume as a consequence of physical aging is responsible for the reduction of the rate of properties change. The initial mobility can be restored by "thermally rejuvenating" the system above $T_{\mathrm{g}}$. Beside this way of reinitiate physical aging, Struik noticed that the application of large stresses above the yield point can produce a similar effect on the mobility of the system as thermal rejuvenation [3]. For this reason the term "mechanical rejuvenation" was used to describe this process. Despite the described effect on the system mobility, several studies showed an increase in the overall density, and therefore in the total free volume, after plastic deformation of polystyrene (PS) [4], polycarbonate (PC) and acrylonitrile-butadiene-styrene copolymer (ABS) [5]. In view of free volume theories, this paradoxical experimental evidence has captured the attention of the scientific community and the idea of an amorphous-amorphous transition was proposed as opposed to the hypothesis of mechanical rejuvenation [6,7]. However, no hint on the microscopic structure of the new reached state and an explanation for the effect on mobility as related to free volume has been given. The aim of this study is to detail the nanoscopic distribution of the free volume after thermal and mechanical rejuvenation and its subsequent time evolution, by means of positron annihilation lifetime spectroscopy (PALS). o-Ps lifetime of the long-living particle is related to the size of the free volume cavity using the following semi-empirical equation [8]:

$$
\tau_{o-\mathrm{Ps}}=\frac{1}{2}\left[1-\frac{R}{R+\Delta R}+\frac{1}{2 \pi} \sin \left(\frac{2 \pi R}{R+\Delta R}\right)\right]^{-1},
$$

where $R$ is the radius of the cavity. Equation (1) describes o-Ps lifetime in an infinitely deep potential well with $\Delta R=1.656 \AA$, a length scale parameter that accounts for the overlap of the positron and the electron wave function.

The intensity of $o$-Ps $\left(I_{o-\mathrm{Ps}}\right)$, which is proportional to the probability of positronium formation, in the past has been related to the concentration of free volume holes in the polymer [9]. However, it was found that $I_{o-\mathrm{Ps}}$ is also affected by a number of other non-structural variables [10-13]. Although the interpretation of $I_{o-P s}$ as a measure for free volume has been questioned [14], we believe that, provided that all the previously mentioned effects are eliminated, a direct relation between $I_{o-P s}$ and the hole density exists. That is the case of our experimental conditions, as shown in the past where the reduction of $I_{o-\mathrm{Ps}}$ during physical aging of PC, gave useful information on the evolution of the polymer structure [15]. For this reason, we use the following equation to evaluate the variation of the free volume percentage occurring after different themo-mechanical treatments: 


$$
\begin{gathered}
\left(\frac{\nu_{\mathrm{f}}}{V_{\text {tot }}}-\frac{\nu_{\mathrm{ff} 0}}{V_{\text {tot. } 0}}\right) \% \cong \frac{\nu_{\mathrm{f}}-\nu_{\mathrm{f} 0}}{V_{\text {tot. } 0}} \%=\Delta f_{\nu} \% \\
=A \frac{4}{3} \pi\left(R^{3} I_{o-\mathrm{Ps}}-R_{0}^{3} I_{o-\mathrm{Ps}(0)}\right),
\end{gathered}
$$

where the subscript (0) indicates the free volume properties of the "fresh" samples, $V_{\text {tot. }}$ is the overall volume and $A$ is an empirical parameter taken from literature data. The value of $R$ is derived from the lifetime using Eq. (1). We assume a linear relation between the concentration of holes and $I_{o-\mathrm{Ps}}$ since the variation of $I_{o-\mathrm{Ps}}$ is small, as it will be seen in the results section.

\section{Experimental}

The materials used in this study are a commercial grade PC (Lexan 161) from General Electric Co. and a commercial grade PS with $M_{w}=318490$ and polydispersity of 3.09. Physical aging after thermal rejuvenation above $T_{\mathrm{g}}$ on $3.2 \mathrm{~mm}$ thick samples was monitored for PC at room temperature. Details on sample preparation can be found in Ref. [15]. "Well-aged" injection moulded samples were employed for plastic deformation by means of cold rolling. The thickness reductions investigated in this study are summarised in the Table. For all thickness reductions, all of the samples pass the yield point and reach the plastic deformation region. PALS measurements were performed at room temperature on all the above-mentioned samples, by exposing them to radioactive ${ }^{22} \mathrm{NaCl}$ with an activity of $10 \mu \mathrm{C}$. Details on the experimental set-up are given elsewhere [15]. Two million counts were collected for each spectrum, which corresponds to an irradiation time of 8 hours that is short enough to avoid any charging effects [11], provided that a "fresh" sample is employed for each experiment. The spectra are analysed using the program POSITRONFIT that describes the spectrum as a convolution of the instrument resolution function and a finite number of negative exponentials plus the background [16].

\section{Results and discussion}

The time evolution of $\tau_{o-\mathrm{Ps}}$ and $I_{o-\mathrm{Ps}}$ during PC physical aging at room temperature after thermal rejuvenation above $T_{\mathrm{g}}$ is shown in Fig. 1. From the observation of this figure, $\tau_{o-P s}$ does not show any variation with aging time, implying no changes in the cavity size, while $I_{o-\mathrm{Ps}}$, related to the concentration of holes, clearly decays as aging proceeds. Analogous results were obtained at other aging temperatures [15].

The effect of the thickness reduction just after cold rolling on $\tau_{o-\mathrm{Ps}}$ and $I_{o-\mathrm{Ps}}$ of PC and PS is shown in the Table. The following observations can be made: (i) Both $\tau_{o-\text { Ps }}$ and $I_{o-\text { Ps }}$ undergo a significant change upon cold rolling in the plastic deformation region. In particular, $\tau_{o-P s}$ increases, whereas a reduction 


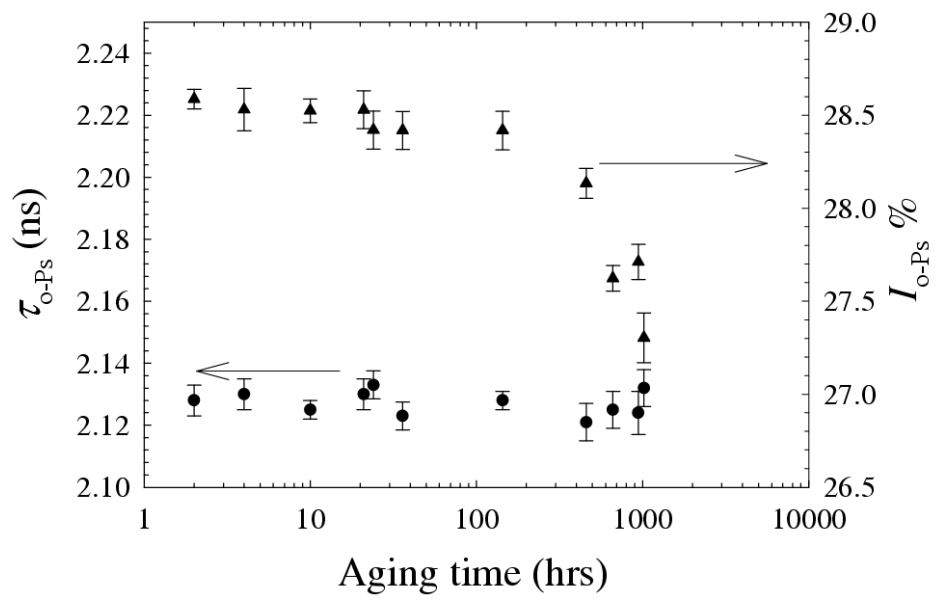

Fig. 1. Time evolution for PC after thermal rejuvenation above $T_{\mathrm{g}}$ of the PALS parameters: $\tau_{o-\mathrm{Ps}}$ (circles), $I_{o-\mathrm{Ps}}$ (triangles).

of $I_{o-P s}$ occurs; (ii) No significant dependence of the PALS parameters on the thickness reduction appears, provided that the samples are rolled above the yield point as done in this study; (iii) PC and PS show a similar trend.

TABLE

$\mathrm{PC}$ and PS percentage of thickness reduction and its effect on $\tau_{o-\mathrm{Ps}}$ and $I_{o-\mathrm{Ps}}$.

\begin{tabular}{c|c|c|c|c|c}
\hline \hline PC thick. red. \% & 0 & 19 & 28 & 33 & 41 \\
\hline$\tau_{o-\mathrm{Ps}}$ & 2.127 & 2.16 & 2.176 & 2.158 & 2.161 \\
$I_{o-\mathrm{Ps}}$ & 29.2 & 27.5 & 27.4 & 27.6 & 17.7 \\
\hline PS thick. red. $\%$ & 0 & 20 & 29 & 35 & 40 \\
\hline$\tau_{o-\mathrm{Ps}}$ & 2.165 & 2.228 & 2.233 & 2.23 & 2.236 \\
$I_{o-\mathrm{Ps}}$ & 34.6 & 33.0 & 32.9 & 33.0 & 32.7
\end{tabular}

Using Eq. (2), with $A=0.00226 \AA^{-3}$ for PC and $A=0.00127 \AA^{-3}$ for PS [17], the free volume change after plastic deformation can be determined. The results, shown in Fig. 2, indicate a free volume change independent of the thickness reduction corresponding to a variation of the specific volume of about $0.2 \%$ for PC. For PS, the free volume does not change significantly, although the microstructure must have changed dramatically as shown by the behaviour of $\tau_{o-\mathrm{Ps}}$ and $I_{o-\mathrm{Ps}}$.

Interesting is also the time evolution of the free volume after plastic deformation shown in Fig. 3. Contrarily to thermally rejuvenated samples, in this case the free volume reduction is fully related to a reduction of $\tau_{o-P s}$ (here not shown), while $I_{o-P s}$ does not present significant changes. Moreover, $\tau_{o-P s}$ tends to assume the value before plastic deformation. 


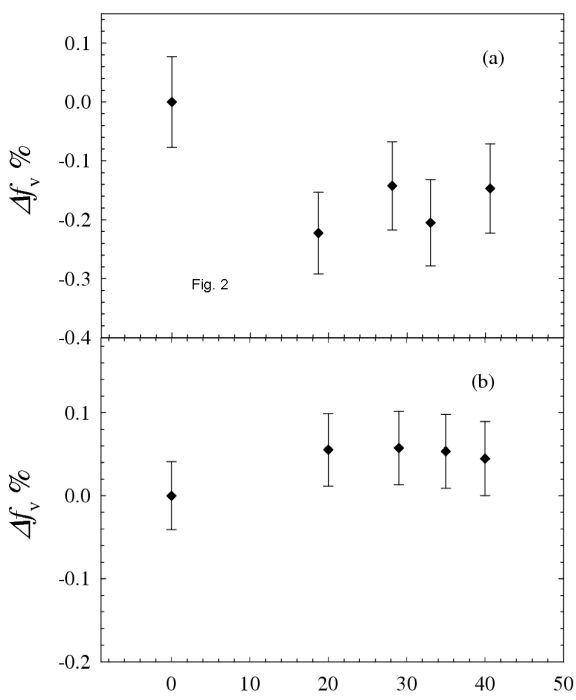

Fig. 2

Thickness reduction $(\%)$

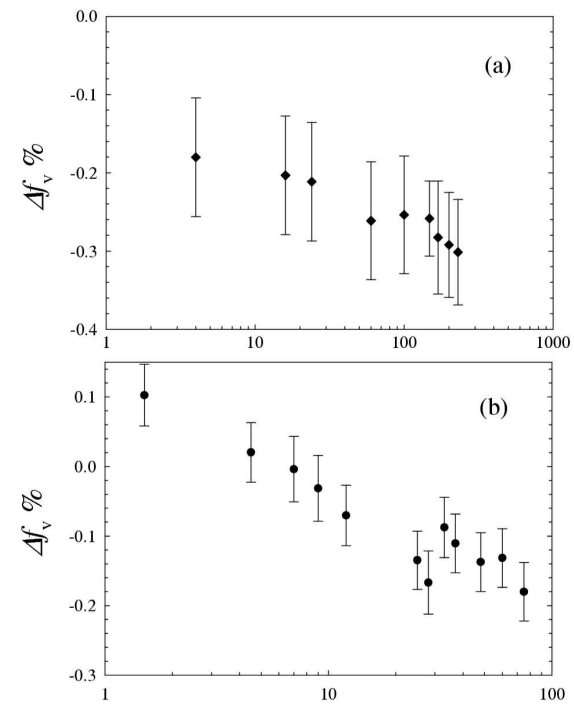

Fig. 3 Aging time (hrs)

Fig. 2. Effect of the percentage of thickness reduction on the variation of the free volume percentage of (a) PC and (b) PS.

Fig. 3. Time evolution after cold rolling of the variation of the free volume percentage of (a) PC and (b) PS.

These results clearly imply that the microstructure assumed by plastically deformed samples and the mechanism of the subsequent structural relaxation are far different from those of thermally rejuvenated samples. Therefore, our present PALS results support the hypothesis of an amorphous-amorphous transition, as proposed by McKenna [6], whereas the idea of rejuvenation induced by mechanical deformation as formulated by Struik [3] is clearly denied. The rejuvenating effect of plastic deformation was misinterpreted due to its ability to reinitiate physical aging similar to thermal rejuvenation. However, the main feature of the time evolution of the free volume after plastic deformation is the reduction of the cavity size, as testified by the reduction of $\tau_{o-\mathrm{Ps}}$, rather than the reduction of the concentration of free volume holes as is the case of physical aging after thermal rejuvenation. In other words, although the macroscopic volume reduction is very similar in both cases, the microscopic mechanism for free volume reduction is totally different. In addition, another important observation of the effect produced by plastic deformation is the persistence of the change of $I_{o-\mathrm{Ps}}$, which can be considered as a signature of the new amorphous state.

Finally, we would like to comment on the interpretation of our PALS results. The reduction of $I_{o-P s}$ upon cold rolling could be an artefact due to the formation of free radicals induced by application of stress. However, we do not observe any time evolution of $I_{o-P s}$ as would be expected if slowly decaying free radicals were 
present in the samples. Therefore, we can exclude any interference on $I_{o-P s}$ coming from free radicals formation. These observations support our interpretation of $I_{o-\mathrm{Ps}}$ as a factor that provides significant information on the polymer structure.

\section{Conclusions}

The effect on free volume of PC and PS of thermal rejuvenation above $T_{\mathrm{g}}$ and plastic deformation and its subsequent time evolution were investigated by means of PALS. Despite the ability of the two treatment to reinitiate physical aging, our results suggest that plastic deformation leads to a totally different microstructure compared to that obtained after thermal rejuvenation. In particular, the former treatment produces an increase in $\tau_{o-\mathrm{Ps}}$ and a decrease in $I_{o-\mathrm{Ps}}$, whereas $\tau_{o-\mathrm{Ps}}$ is not affected by the thermal rejuvenation and $I_{o-P s}$ experiences an increase. In addition, the subsequent time evolution consists of a reduction of $\tau_{o-\mathrm{Ps}}$, which tends to return to its original value, and a constant $I_{o-\text { Ps }}$ for plastically deformed samples. On the other hand, the structural relaxation of thermally rejuvenated samples consists of a reduction of $I_{o-\mathrm{Ps}}$. These results support the hypothesis of an amorphous-amorphous transition induced by plastic deformation.

\section{References}

[1] D. Turnbull, M.H. Cohen, J. Chem. Phys. 34, 120 (1961).

[2] A.J. Kovacs, Adv. Polym. Sci. 3, 394 (1963).

[3] L.C.E. Struik, Physical Aging in Amorphous Glassy Polymers and Other Materials, Elsevier Science, Amsterdam 1978.

[4] H.G.H. Van Melick, L.E. Govaert, B. Raas, W.J. Nauta, H.E.H. Meijer, Polymer 44, 1171 (2003).

[5] J.L. Broutman, R.S. Patil, Polym. Eng. Sci. 11, 165 (1971).

[6] G.B. McKenna, J. Phys., Condens. Matter 15, 737 (2003).

[7] D.L. Malandro, D.J. Lacks, J. Chem. Phys. 110, 4593 (1999); D.J. Lacks, Phys. Rev. Lett. 84, 4629 (2000).

[8] M. Eldrup, D. Lightbody, J.N. Sherwood, Chem. Phys. 63, 51 (1981).

[9] V.P. Shantarovich, J. Radioanal. Nucl. Chem. 210, 357 (1996).

[10] C. Wastlund, F.H.J. Maurer, Polymer 39, 2897 (1998).

[11] M. Welander, F.H.J. Maurer, Mater. Sci. Forum 105-110, 1811 (1992); D. Cangialosi, H. Schut, M. Wübbenhorst, J. van Turnhout, A. van Veen, Radiat. Phys. Chem. 68, 507 (2003).

[12] C.L. Wang, K. Hirata, J. Kawahara, Y. Kobayashi, Phys. Rev. B 58, 14864 (1998); A. Alba García, L.D.A. Siebbeles, H. Schut, A. van Veen, Radiat. Phys. Chem. 68, 515 (2003).

[13] J. Zrubcova, J. Kristiak, W.B. Pedersen, N.J. Pedersen, M. Eldrup, Mater. Sci. Forum 363, 359 (2001). 
[14] F.H.J. Maurer, M. Schmidt, Radiat. Phys. Chem. 58, 509 (2000).

[15] D. Cangialosi, H. Schut, A. van Veen, S.J. Picken, Macromolecules 36, 142 (2003); D. Cangialosi, M. Wübbenhorst, H. Schut, A. van Veen, S.J. Picken, Phys. Rev. B 69, 134206 (2004).

[16] P. Kirkegaard, M. Eldrup, O.E. Mogensen, N.J. Pedersen, Comp. Phys. Commun. 23, 307 (1981)

[17] K. Hagiwara, T. Ougizawa, T. Inoue, K. Hirata, Y. Kobayashi, Radiat. Phys. Chem. 58, 525 (2000). 